\title{
Classification of potential pathways affecting the VEGF pathway in glioblastoma multiforme (GBM) recurrent: A bioinformatics study based on differentially expressed- microRNAs
}

\section{Morteza Hadizadeh}

Kerman University of Medical Sciences

\section{Ramin Soltani}

Shahrekord University of Medical Sciences

Taimour Langaee

University of Florida

Marziye Shad pirouz

Department of Applied Matemathics, Faculty of Mathematical Sciences, Shahrood University of Technology, Semnan

\section{R. Mozafari}

Supreme NanoBiotics Co. Ltd. and Supreme Pharmatech Co. Ltd

Sorayya Ghasemi ( $\sim$ sorayya.ghasemi@gmail.com )

Shahrekord University of Medical Sciences

\section{Research Article}

Keywords: VEGF, GBM-recurrent, angiogenesis, signaling pathways, GSE32466, bioinformatics approach

Posted Date: July 8th, 2021

DOI: https://doi.org/10.21203/rs.3.rs-677315/v1

License: (c) (i) This work is licensed under a Creative Commons Attribution 4.0 International License. Read Full License 


\section{Abstract}

Glioblastoma multiforme (GBM) resistance to anti-angiogenesis drugs results in recurrence of the disease which leads to death. The resistance to anti-angiogenesis drugs that target the VEGF pathway is due to the influence of other pathways. This study aimed to identify and classify the pathways that are related to the VEGF pathway in GBM recurrent. The identification of differentially expressed miRNAs (DEmiRNAs) based on GBM GSE profiles (GSE32466) were carried out using a LIMMA R package and VEGF pathway genes in the KEGG database. Pathways related to DEmiRNAs and VEGF pathway genes were discovered by DIANA-miRPath v3.0, NetworkAnalyst and ToppGene databases, respectively. Inhibitory or activity affecting pathways relating to VEGF pathway were obtained based on XTalkDB database. The classification was determined by the KEGG database. There were 1014 genes that were found to have interaction with VEGF signaling pathway genes. One hundred ninteen pathways were achieved which have overlapping genes with the VEGF pathway genes. The MAPK pathway had the most in common genes with the VEGF pathway (39 genes). A total of 91 pathways were identified in 24 different classes. Several pathways significantly affect the VEGF pathway. Hence, it seems necessary to achieve new targets for combination therapies for GBM.

\section{Introduction}

Glioblastoma multiforme (GBM), the most aggressive CNS tumor with the poorest prognosis, is caused by extreme and abnormal angiogenesis ${ }^{[1,2]}$. In spite of multiple aggressive management of GBM, the outcome is unpleasant and patients generally die of tumor recurrences ${ }^{[3]}$. Angiogenesis is considered one of the main hallmarks of GBM and for this reason, this type of tumor is an attractive candidate for anti-angiogenic therapies, especially during recurrence ${ }^{[4]}$. The angiogenesis phenomenon occurs through a variety of modalities mainly vascular endothelial growth factor (VEGF), and other members of the VEGF family play important roles as well. This has signified the basis for the development of anti-VEGF blocking mediators and their use in GBM treatment. Nevertheless, these agents failed to induce significant therapeutic effects ${ }^{[5]}$. Bevacizumab (BEV), a monoclonal antibody against VEGF, has only transient effects and GBM recurrence is not avoidable ${ }^{[6,7]}$. Increasing information about GBMepigenetics help increase our knowledge related to the tumorigenesis, development, and recurrence of $\mathrm{GBM}^{[8]}$. The interactions between epigenetic factors such as miRNAs and the cell signaling pathways are considered as high-potential targets for new therapeutic approaches ${ }^{[9]}$. Several miRNAs can modulate mRNAs, thus generating an extraordinary complex regulatory network involved in many pathways such as drug resistance, angiogenesis, and recurrence ${ }^{[9-12]}$. A literature survey of a set of miRNA deregulated in GBM found that they were overexpressed or downmodulated compared to normal brain tissue ${ }^{[9,13]}$. High throughput technologies, including microarrays or next-generation sequencing methods, provided great deal of data that increased our insights into the roles of non-coding RNAs (ncRNAs) and microRNAs [14] 
The purpose of this in silico study was to identify pathways that affect VEGF pathway which may cause anti-angiogenesis drugs resistance during recurrence. As one of the recurrence phenomena is angiogenesis, this database has been used to find indirect pathways affecting GBM angiogenesis. It seems that targeting the VEGF pathway may lead to alternative pathways activation and protection of the main pathway functions. In this systems biology analysis, we tried to identify other pathways that triger this phenomenon and can affect recurrence via their effect on the VEGF pathway. By categorizing these pathways, we may provide better synergistic therapies with existing drugs for VEGF pathway targeting along with drugs that target other effective pathways. This may help eliminate resistance to antiangiogenesis therapies in GBM possibly by overcoming its recurrence.

\section{Results}

\subsection{Data collection: Identification of DEmiRNAs}

The summarized of this in silico analysis is including: One microarray miRNA expression dataset of GSE32466 (GPL10850) was used in this study. The dataset contains 24 samples (miRNA expression profiles between primary and recurrent GBM tissues from the same patients).

After data normalization and quality control between GBM $(n=12)$ and recurrent GBM samples $(n=12)$ we identified 79 DEmiRNAs: 30 up-regulated miRNAs and 49 down-regulated miRNAs (Supplementary File 1). Then DEmiRNAs were used for cluster analysis, the pheatmap package from $R$ software was used for hierarchical cluster analysis of DEmiRNAs, as shown in Fig. 1.

\subsection{Data collection: VEGF signaling pathway genes}

Using KEGG PATHWAY Database (https://www.genome.jp/kegg/pathway.html), all genes $(n=59)$ in the VEGF signaling pathway were collected. As shown in Table 1 gene expression of some of these genes were difference between GBM and normal brain tissue. According to the Expression Atlas site, the expression of 14 genes out of 59 genes in the VEGF signaling pathway showed increasedexpression, and 37 genes showed decreased expression (adjusted $p$ value $\leq 0.05$ ). For the remaining genes, no changes in expression were observed. 
Table 1

VEGF signaling pathway genes: Changes in the expression levels of VEGF pathway genes reported in this table are listed based on the Expression Atlas site (www.ebi.ac.uk/gxa) for GBM Vs. normal. Up: upregulation, Down: down-regulation, Unknown:There is no report in the Expression Atlas site.

\begin{tabular}{|c|c|c|c|}
\hline & Symbol & Description & Expression \\
\hline 1 & VEGFA & vascular endothelial growth factor $A$ & Up \\
\hline 2 & HSPB1 & heat shock protein family B (small) member 1 & Up \\
\hline 3 & SPHK1 & sphingosine kinase 1 & Up \\
\hline 4 & RAC2 & Rac family small GTPase 2 & Up \\
\hline 5 & PXN & paxillin & Up \\
\hline 6 & NRAS & NRAS proto-oncogene, GTPase & Up \\
\hline 7 & RAF1 & Raf-1 proto-oncogene, serine/threonine kinase & Up \\
\hline 8 & KDR & kinase insert domain receptor & Up \\
\hline 9 & PLA2G4A & phospholipase A2 group IVA & Up \\
\hline 10 & SH2D $2 A$ & $\mathrm{SH} 2$ domain containing $2 \mathrm{~A}$ & Up \\
\hline 11 & MAPKAPK3 & MAPK activated protein kinase 3 & Up \\
\hline 12 & MAPKAPK2 & MAPK activated protein kinase 2 & Up \\
\hline 13 & CDC42 & cell division cycle 42 & Up \\
\hline 14 & NFATC2 & nuclear factor of activated T cells 2 & Up \\
\hline 15 & PRKCA & protein kinase $\mathrm{C}$ alpha & Down \\
\hline 16 & MAPK11 & mitogen-activated protein kinase 11 & Down \\
\hline 17 & AKT3 & AKT serine/threonine kinase 3 & Down \\
\hline 18 & PIK3CD & $\begin{array}{l}\text { phosphatidylinositol-4,5-bisphosphate 3-kinase catalytic } \\
\text { subunit delta }\end{array}$ & Down \\
\hline 19 & MAP2K2 & mitogen-activated protein kinase kinase 2 & Down \\
\hline 20 & RAC3 & Rac family small GTPase 3 & Down \\
\hline 21 & MAP2K1 & mitogen-activated protein kinase kinase 1 & Down \\
\hline 22 & PIK3CB & $\begin{array}{l}\text { phosphatidylinositol-4,5-bisphosphate 3-kinase catalytic } \\
\text { subunit beta }\end{array}$ & Down \\
\hline 23 & HRAS & HRas proto-oncogene, GTPase & Down \\
\hline 24 & PIK3R1 & phosphoinositide-3-kinase regulatory subunit 1 & Down \\
\hline
\end{tabular}




\begin{tabular}{|c|c|c|c|}
\hline & Symbol & Description & Expression \\
\hline 25 & PIK3R3 & phosphoinositide-3-kinase regulatory subunit 3 & Down \\
\hline 26 & Nos3 & nitric oxide synthase 3 & Down \\
\hline 27 & PLCG2 & phospholipase $\mathrm{C}$ gamma 2 & Down \\
\hline 28 & MAPK1 & mitogen-activated protein kinase 1 & Down \\
\hline 29 & PRKCG & protein kinase $\mathrm{C}$ gamma & Down \\
\hline 30 & SPHK2 & sphingosine kinase 2 & Down \\
\hline 31 & PLCG1 & phospholipase $\mathrm{C}$ gamma 1 & Down \\
\hline 32 & PPP3CC & protein phosphatase 3 catalytic subunit gamma & Down \\
\hline 33 & MAPK12 & mitogen-activated protein kinase 12 & Down \\
\hline 34 & PLA2G4D & phospholipase A2 group IVD & Down \\
\hline 35 & AKT2 & AKT serine/threonine kinase 2 & Down \\
\hline 36 & PIK3R2 & phosphoinositide-3-kinase regulatory subunit 2 & Down \\
\hline 37 & PPP3CA & protein phosphatase 3 catalytic subunit alpha & Down \\
\hline 38 & PLA2G4C & phospholipase A2 group IVC & Down \\
\hline 39 & PLA2G4F & phospholipase A2 group IVF & Down \\
\hline 40 & PLA2G4E & phospholipase A2 group IVE & Down \\
\hline 41 & $\mathrm{SHC} 2$ & SHC adaptor protein 2 & Down \\
\hline 42 & PLA2G4B & phospholipase A2 group IVB & Down \\
\hline 43 & $\begin{array}{l}\text { JMJD7- } \\
\text { PLA2G4B }\end{array}$ & JMJD7-PLA2G4B readthrough & Down \\
\hline 44 & РPР3СВ & protein phosphatase 3 catalytic subunit beta & Down \\
\hline 45 & PTGS2 & prostaglandin-endoperoxide synthase 2 & Down \\
\hline 46 & PPP3R2 & protein phosphatase 3 regulatory subunit $B$, beta & Down \\
\hline 47 & PRKCB & protein kinase $\mathrm{C}$ beta & Down \\
\hline 48 & PPP3R1 & protein phosphatase 3 regulatory subunit $B$, alpha & Down \\
\hline 49 & PTK2 & protein tyrosine kinase 2 & Down \\
\hline 50 & KRAS & KRAS proto-oncogene, GTPase & Down \\
\hline 51 & MAPK3 & mitogen-activated protein kinase 3 & Down \\
\hline
\end{tabular}




\begin{tabular}{|llll|}
\hline & Symbol & Description & Expression \\
\hline 52 & SRC & SRC proto-oncogene, non-receptor tyrosine kinase & Unknown \\
\hline 53 & MAPK13 & mitogen-activated protein kinase 13 & Unknown \\
54 & BAD & BCL2 associated agonist of cell death & Unknown \\
\hline 55 & MAPK14 & mitogen-activated protein kinase 14 & Unknown \\
56 & AKT1 & AKT serine/threonine kinase 1 & Unknown \\
57 & CASP9 & caspase 9 & Unknown \\
58 & PIK3CA & $\begin{array}{l}\text { phosphatidylinositol-4,5-bisphosphate 3-kinase catalytic } \\
\text { subunit alpha }\end{array}$ & Unknown \\
\hline 59 & RAC1 & Rac family small GTPase 1 & Unknown \\
\hline
\end{tabular}

\subsection{Pathways related to DEmiRNAs detection}

DIANA-miRPath v.3 software was used to find the biological significant pathways of the 79 DEmiRNAs. The DEmiRNAs were differentially expressed between the primary GBM and recurrent GBM. The analysis revealed that 49 groups enriched-pathways were significantly related to 22 up-regulated miRNAs and 29 down-regulated miRNAs. Among up-regulated DEmiRNAs and according to the KEGG pathway maps, hsamiR-497-5p was present in most pathways (34 pathways), and hsa-miR-204-5p and hsa-miR-551b-3p, in contrast, participated in only one pathway. On the other hand, 57 pathways were related to hsa-miR-17-5p (the most involved pathway) but only 2 pathways were related to hsa-miR-31-3p (the least involved pathway) in down-regulated DEmiRNAs (Supplementary File 2a, 2b).

\subsection{Genes and pathways related to the VEGF signaling pathway genes}

Interactional genes (1014 genes) were found to have interaction with 59 genes of the VEGF signaling pathway by NetworkAnalyst (Supplementary File 3). In addition, all significant pathways of interactional genes were identified and according to the enrichment analysis 170 significant pathways were recognized for interactional genes by ToppGene. Cancer Pathways had the most interactional genes (180 genes); while, Sphingosine degradation had the fewest interactional genes (3 genes) (Supplementary File 4). Moreover, 119 pathways were achieved by NetworkAnalyst, some of their genes overlaped with the VEGF signaling pathway. The MAPK signaling pathway had the most in common with the VEGF signaling pathway (39 genes). Alpha-Linolenic acid metabolism, Linoleic acid metabolism, Thyroid cancer, Ether lipid metabolism, Adherens junction and IL-17 signaling pathways had the least gene overlapping with VEGF signaling pathway (7 genes) (Supplementary File 5).

\subsection{Crosstalk analysis}


XTalkDB (http://www.xtalkdb.org/home) demonstrated all pathways that inhibited or activated VEGF signaling pathway. Some pathways had inhibition and activation effects (dual effects) on the VEGF signaling pathway, such as Neurotrophin signaling pathway, Notch signaling pathway and Progesteronemediated oocyte maturation. In contrast, some pathways had only one-way effect (activation or inhibition) on the VEGF signaling pathway. Reciprocally, the VEGF signaling pathway activated or inhibited some pathways. All crosstalks are shown in Fig. 2.

\subsection{Common miRs between VEGF pathway and DE-miRNAs}

The eight miRNAs obtained (including hsa-miR-204-5p, hsa-miR-17-5p, hsa-miR-195-5p, hsa-miR-497-5p, hsa-let-7a-5p, hsa-miR-221 -3p, hsa-miR-26b-5p, hsa-miR-21-5p) were common in both the VEGF signaling pathway and DEmiRNAs in the GSE32466 dataset (supplementary file 7).

\subsection{Identification of classes for final pathways}

Class assessment of all finalized pathways was performed by the KEGG PATHWAY database. All 91 finalized pathways were eventually classified into 24 different classes. "Signal transduction" and "Cancer: specific types" are the largest classes in this study. Fifteen pathways were related to "Signal transduction". Some of these pathways were involved in angiogenesis process such as Hippo, TGF-beta, TNF, mTOR, and othersignaling pathways. Moreover, 20 pathways were involved in "Cancer: specific types" and cancer overview classes. On the other hand, "Cell motility" and "Circulatory system", and others were the lowest classes determined in this study. These classes were common regulator of actin cytoskeleton and adrenergic signaling in cardiomyocytes pathways. Details of the assigned classes to the final related pathways are shown in Table 2 and Fig. 3. The classification shown in Fig. 3 is based on the frequency of related signaling pathways. Table 2 also shows that the classes we obtained in this study are partly in accord with other studies. 
Table 2

The final pathways obtained from the analysis steps based on the KEGG PATHWAY site. Some classes are more diverse in terms of signaling pathways. Based on an evidence, some classes have functions associated with certain phenotypes in GBM or other cancers. For some that are not mentioned in the reference table, there is no relevant reference yet.

\begin{tabular}{|c|c|c|c|c|}
\hline No. & Classes & Related signaling pathways & $\begin{array}{l}\text { classes or Pathways } \\
\text { functions in GBM or } \\
\text { cancers }\end{array}$ & Ref. \\
\hline \multirow[t]{15}{*}{1} & \multirow{15}{*}{$\begin{array}{l}\text { Signal } \\
\text { transduction }\end{array}$} & - MAPK signaling pathway & \multirow{15}{*}{$\begin{array}{l}\text { Signaling pathways are } \\
\text { most affected by } \\
\text { mutations and } \\
\text { epigenetic changes in } \\
\text { cancer cells and are } \\
\text { involved in essential } \\
\text { GBM hallmarks. }\end{array}$} & \multirow{15}{*}{$\begin{array}{l}{[26,27,} \\
45]\end{array}$} \\
\hline & & • ErbB signaling pathway & & \\
\hline & & • PI3K-Akt signaling pathway & & \\
\hline & & - Sphingolipid signaling pathway & & \\
\hline & & - FoxO signaling pathway & & \\
\hline & & - mTOR signaling pathway & & \\
\hline & & • Wnt signaling pathway & & \\
\hline & & - HIF-1 signaling pathway & & \\
\hline & & • TNF signaling pathway & & \\
\hline & & • AMPK signaling pathway & & \\
\hline & & $\begin{array}{l}\text { - Phosphatidylinositol signaling } \\
\text { system }\end{array}$ & & \\
\hline & & • Hippo signaling pathway & & \\
\hline & & - TGF-beta signaling pathway & & \\
\hline & & - VEGF signaling pathway & & \\
\hline & & - NF-kappa B signaling pathway & & \\
\hline \multirow[t]{2}{*}{2} & \multirow[t]{2}{*}{ Cancer } & $\begin{array}{l}\text { - specific types: (Prostate cancer, } \\
\text { Chronic myeloid leukemia, } \\
\text { Glioma, Pancreatic cancer, Renal } \\
\text { cell carcinoma, Non-small cell } \\
\text { lung cancer, Colorectal cancer, } \\
\text { Acute myeloid leukemia, } \\
\text { Endometrial cancer, Small cell } \\
\text { lung cancer, Melanoma, Bladder } \\
\text { cancer, Thyroid cancer) }\end{array}$ & \multirow[t]{2}{*}{$\begin{array}{l}\text { In addition to the } \\
\text { unique changes, there } \\
\text { are common pathways } \\
\text { involved in GBM and } \\
\text { other cancers. }\end{array}$} & \multirow[t]{2}{*}{ [46] } \\
\hline & & $\begin{array}{l}\text { - Overview: (Pathways in cancer, } \\
\text { Proteoglycans in cancer, Viral } \\
\text { carcinogenesis, Central carbon } \\
\text { metabolism in cancer, Choline } \\
\text { metabolism in cancer, MicroRNAs } \\
\text { in cancer, Transcriptional } \\
\text { misregulation in cancer) }\end{array}$ & & \\
\hline
\end{tabular}




\begin{tabular}{|c|c|c|c|c|}
\hline No. & Classes & Related signaling pathways & $\begin{array}{l}\text { classes or Pathways } \\
\text { functions in GBM or } \\
\text { cancers }\end{array}$ & Ref. \\
\hline 3 & Endocrine system & $\begin{array}{l}\text { - Insulin signaling pathway } \\
\text { - Thyroid hormone signaling } \\
\text { pathway } \\
\text { - Estrogen signaling pathway } \\
\text { - Progesterone-mediated oocyte } \\
\text { maturation } \\
\text { - Oxytocin signaling pathway } \\
\text { - Prolactin signaling pathway } \\
\text { - Adipocytokine signaling } \\
\text { pathway } \\
\text { - Thyroid hormone synthesis }\end{array}$ & $\begin{array}{l}\text { Various studies have } \\
\text { observed the roles and } \\
\text { effect of the endocrine } \\
\text { system on GBM cell } \\
\text { phenotypes. }\end{array}$ & $\begin{array}{l}{[29-33,} \\
47,48]\end{array}$ \\
\hline 4 & Infectious disease & $\begin{array}{l}\text { - Bacterial (Bacterial invasion of } \\
\text { epithelial cells, Shigellosis, } \\
\text { Epithelial cell signaling in } \\
\text { Helicobacter pylori infection, } \\
\text { Salmonella infection, Pathogenic } \\
\text { Escherichia coli infection, } \\
\text { Legionellosis) } \\
\text { - Viral (Hepatitis B, HTLV-I } \\
\text { infection, Epstein-Barr virus } \\
\text { infection, Hepatitis C, Measles) } \\
\text { - Parasitic (Malaria, Chagas } \\
\text { disease (American } \\
\text { trypanosomiasis)) }\end{array}$ & $\begin{array}{l}\text { To date, there is no } \\
\text { study linking GBM to } \\
\text { the causes of infections } \\
\text { other than the virus. }\end{array}$ & [34] \\
\hline 5 & $\begin{array}{l}\text { Cellular } \\
\text { community - } \\
\text { eukaryotes }\end{array}$ & $\begin{array}{l}\text { - Focal adhesion } \\
\text { - Adherens junction } \\
\text { - Gap junction } \\
\text { - Signaling pathways regulating } \\
\text { pluripotency of stem cells }\end{array}$ & $\begin{array}{l}\text { roles of the cellular } \\
\text { community are related } \\
\text { to the characteristics of } \\
\text { invasion, migration, and } \\
\text { apoptosis of GBM cells }\end{array}$ & {$[35,49-51]$} \\
\hline 6 & $\begin{array}{l}\text { Cell growth and } \\
\text { death }\end{array}$ & $\begin{array}{l}\cdot \text { Apoptosis } \\
\text { - Cell cycle } \\
\text { - p53 signaling pathway } \\
\text { - Oocyte meiosis }\end{array}$ & $\begin{array}{l}\text { Important pathways } \\
\text { such as cell cycle and } \\
\text { apoptosis are involved } \\
\text { in the basic hallmarks } \\
\text { of GBM that are } \\
\text { associated with } \\
\text { resistance to present } \\
\text { treatment. }\end{array}$ & {$[52-55]$} \\
\hline
\end{tabular}




\begin{tabular}{|c|c|c|c|c|}
\hline No. & Classes & Related signaling pathways & $\begin{array}{l}\text { classes or Pathways } \\
\text { functions in GBM or } \\
\text { cancers }\end{array}$ & Ref. \\
\hline 7 & $\begin{array}{l}\text { Neurodegenerative } \\
\text { disease }\end{array}$ & $\begin{array}{l}\text { - Parkinson's disease } \\
\text { - Huntington's disease } \\
\text { - Prion diseases }\end{array}$ & $\begin{array}{l}\text { There is little evidence } \\
\text { between the biomaker } \\
\text { of some of these } \\
\text { pathways and GBM. }\end{array}$ & [36] \\
\hline 8 & Immune system & $\begin{array}{l}\text { - Platelet activation } \\
\text { - Leukocyte transendothelial } \\
\text { migration } \\
\text { - B cell receptor signaling } \\
\text { pathway }\end{array}$ & $\begin{array}{l}\text { The immune system of } \\
\text { the CNS plays an } \\
\text { important role in the } \\
\text { biological processes of } \\
\text { GBM, such as } \\
\text { angiogenesis. }\end{array}$ & {$[37,38]$} \\
\hline 9 & Nervous system & $\begin{array}{l}\text { - Neurotrophin signaling pathway } \\
\text { - Long-term depression }\end{array}$ & $\begin{array}{l}\text { Neurotrophic factors } \\
\text { (NTFs) are the most } \\
\text { well-known factor } \\
\text { common between the } \\
\text { Nervous system and } \\
\text { GBM. Stress and } \\
\text { lifestyle may also be } \\
\text { linked to GBM patients' } \\
\text { longevity through this } \\
\text { class. }\end{array}$ & [56-58] \\
\hline 10 & $\begin{array}{l}\text { Cardiovascular } \\
\text { disease }\end{array}$ & $\begin{array}{l}\text { - Arrhythmogenic right ventricular } \\
\text { cardiomyopathy (ARVC) } \\
\text { - Viral myocarditis }\end{array}$ & $\begin{array}{l}\text { There are no studies on } \\
\text { this relationship for } \\
\text { humans. }\end{array}$ & [59] \\
\hline 11 & $\begin{array}{l}\text { Development and } \\
\text { regeneration }\end{array}$ & $\begin{array}{l}\text { - Dorso-ventral axis formation } \\
\text { - Axon guidance }\end{array}$ & $\begin{array}{l}\text { The role of the axon } \\
\text { guidance pathway has } \\
\text { been identified in GBM } \\
\text { invasion. }\end{array}$ & [60] \\
\hline 12 & $\begin{array}{l}\text { Folding, sorting } \\
\text { and degradation }\end{array}$ & $\begin{array}{l}\text { - Protein processing in } \\
\text { endoplasmic reticulum } \\
\text { - Ubiquitin mediated proteolysis }\end{array}$ & $\begin{array}{l}\text { There is evidence for } \\
\text { the role of } \\
\text { ubiquitination and the } \\
\text { endoplasmic reticulum } \\
\text { in GBM progression. }\end{array}$ & {$[39,61]$} \\
\hline 13 & $\begin{array}{l}\text { Transport and } \\
\text { catabolism }\end{array}$ & $\begin{array}{l}\text { - Endocytosis } \\
\text { - Phagosome }\end{array}$ & $\begin{array}{l}\text { There are examples of } \\
\text { these two pathways in } \\
\text { GBM. }\end{array}$ & {$[62,63]$} \\
\hline 14 & Cell motility & - Regulation of actin cytoskeleton & $\begin{array}{l}\text { Lack of regulation of } \\
\text { actin cytoskeleton is } \\
\text { effective in cell } \\
\text { migration and } \\
\text { angiogenesis of GMB. }\end{array}$ & {$[64,65]$} \\
\hline
\end{tabular}




\begin{tabular}{|lllll|}
\hline No. & Classes & Related signaling pathways & $\begin{array}{l}\text { classes or Pathways } \\
\text { functions in GBM or } \\
\text { cancers }\end{array}$ & Ref. \\
\hline 15 & Circulatory system & $\begin{array}{l}\text { - Adrenergic signaling in } \\
\text { cardiomyocytes }\end{array}$ & $\begin{array}{l}\text { Activation of } \beta \text { - } \\
\text { adrenoceptors causes } \\
\text { cell proliferation in } \\
\text { GBM. }\end{array}$ & [66] \\
\hline
\end{tabular}

\section{Discussion}

In silico studies are essential to finding new pathways and genes involved in biological processes. This study used the GSE32466 data set, which includes both recurrent and primary GBM groups. GBM angiogenesis plays an essential role in its recurrence, and the GSE32466 data set is the best available dataset containing data on GBM recurrence ${ }^{[3]}$. Our main goal was to identify classes of indirect pathways that induce VEGF in GBM recurrence. Further understanding of GBM neoangiogenesis may improve our understanding about mechanisms of resistance to anti-angiogenetic drugs. The development of multiomics approaches has led to an impressive deal of new information on a variety of mechanisms and created the opportunity to target GBM selectively ${ }^{[9]}$. In this study, by identifying the miR-DEGs, we found the pathways they target. These pathways seem to be involved in the recurrence of GBM and may also include pathways associated with angiogenesis. The most well-known angiogenic pathway currently treated in cancer therapy is the VEGF signaling pathway. Therefore, we chose the VEGF pathway as our focus hoping that by simultaneously targeting multiple pathways affecting angiogenesis, we may improve the chances of effectively controlling angiogenesis in cancer therapy ${ }^{[15]}$. Studies showed that, there are other signaling pathways affecting the function of the VEGF pathway. For example, a study has shown that glioma cells produce TGF- $\beta$ superfamily ligands which bind TGF- $\beta$ receptors (TGF- $\beta R$ ). In other study, Sui et al. found that VEGF-mediated angiogenesis was activated in the normoxia mode by the TGF- $\beta 1$ / $\beta$-catenin / TCF3 / LEF1 signaling pathway, and in the hypoxia by the HIF-1a / $\beta$-catenin / TCF3 / LEF1 signaling pathway $\left.{ }^{[16} 17\right]$. Therefore, the TGF- $\beta$ pathway can be one of the pathways affecting the function of the VEGF pathway.

Other studies have shown that the HGF/c-MET signaling pathway plays an important role in angiogenesis and tumor growth. Hence, the function of this signaling pathway is synergistic with the VEGF/VEGFR signaling pathway, for instance they have a greater stimulating effect on angiogenesis and tumor growth when treated simultaneously ${ }^{[18,19]}$. Accordingly, the purpose of our study was in silico identification of the pathways with the potential to target the VEGF signaling pathway in recurrent GBM indirectly. In this study, the pathways with overlapping genes or pathways that indirectly affect the VEGF signaling pathway affecting GBM recurrence, were investigated. The VEGF/R targeting therapies are designed to treat GBM angiogenesis; however, the clinical effect of these therapies is limited due to the emergence of drug resistance during treatment ${ }^{[4]}$. The mechanisms underlying cancer drug resistance is still not fully understood. Therefore, it is critical to discover the mechanisms of drug resistance to increase the success rate of GBM treatment, which is a recurrent disease ${ }^{[20,21]}$. In recurrent GBM, 
chromosomal instability (CIN) is considered an important mechanism leading to tumor heterogeneity. In addition, high heterogeneity affects the disregulation of microRNA levels and, consequently, their related functions ${ }^{[9]}$. In this study, we reached the effective pathways through DEmiRNAs in recurring GBM. Based on our findings, among 79 significant DEmiRNAs, eight miRNAs overlapped with the VEGF pathway. Therefore, it seems that if other microRNAs play any role in angiogenesis, they may also indirectly affect the VEGF pathway. Indirect effects may occur in the form of connection or overlap, both of which were examined using NetworkAnalyst. Zhang et al. found that, the anticancer drug convallatoxin induces apoptosis in colon cancer cells by cross-linking the JAK2/STAT3 and mTOR/STAT3 signaling pathways and inhibiting angiogenesis ${ }^{[22]}$. Bo Zhang et al. showed that increasing LncRNA ANRIL expression caused the overexpression of the VEGF gene and induced angiogenesis by activating the NF-KB signaling pathway in a mouse model of diabetes mellitus with stroke ${ }^{[23]}$. Another study found that activation of the Jagged $1 /$ Notch 1 signaling pathway by $17 \beta$-esteradiol is associated with angiogenic factors that may turn tumor cells into angiogenic phenotypes ${ }^{[24]}$. In the study by Nabors et al., it was shown that, extracellular signals such as hypoxia and cytokines induced over-expression of angiogenic factors in glioma cells ${ }^{[25]}$.

GBM cells are involved in many mutations that significantly affect cancerous processes. These mutations lead to low survival rates by increasing the complexity of treatment and resistance to therapy in patients. Different pathways in the intracellular signaling network are dis-regulated due to mutations $[26,27]$. Our study and others also showed that signal transduction pathways are the main pathways that affect angiogenesis. In a functional study in a breast cancer cell line, Zhang et al. showed that the MAPK signaling pathway plays a role in regulating angiogenesis. In this pathway, HGF/SF, together with its receptor (C-MET), causes angiogenesis by increasing VEGF gene expression ${ }^{[28]}$. Twelve percent of the pathways identified and examined in our study affect other cancers as well. In our study, various endocrine systems were identified as pivotal pathways indirectly related to the VEGF pathway. Various studies have emphasized the role and impact of these pathways on the patients, survival, cancer development, and migration of cancer cells and the recurrence of GBM, thus affecting GBM patient survival rates ${ }^{[29,30]}$. Consistent with this study, the role of steroid hormones, prolactin (PRL) and its receptor (PRLR), and leptin-related signaling in GBM has been identified in some functional studies, making them potential therapeutic targets for future studies ${ }^{[31-33]}$. The role of oncoviruses in various cancers has also been identified. There are not many studies on the role of viruses in GBM, but McFalineFigueroa et al., has pointed to the presence of cytomegalovirus in GBM ${ }^{[34]}$. Another class of pathway identified in our in silico analysis is the eukaryotic community. This class includes signaling pathways mostly associated with invasion and apoptosis in cancers, including those in GBM ${ }^{[35]}$.

Interestingly, some of the final pathways we identified are shared between GBM and some diseases and processes not related to cancer. Some of these pathways are associated with neurodegenerative disease, cardiovascular disease, endocrine and metabolic disease, and the digestive system. Perhaps the reason for their apparent irrelevance is the absence of studies investigating the correlation or the common factors between them and GBM. However, in some neurodegenerative diseases, such as prions, a link 
between the prion protein ( $\mathrm{PrPC}$ ) and GBM has been observed ${ }^{[36]}$. The immune system is another class identified in our study. GBM has mechanisms that can help it escape the central nervous system (CNS) immune system and promote some of its own processes, such as angiogenesis ${ }^{[37,38]}$. Some treatments are also being considered in the relapse phase; folding, sorting, degradation, and transport are processes that have not received much attention in cancer studies. However, one study has suggested the role of UBE3C in ANXA7 ubiquitination in the progression of glioma ${ }^{[39]}$. Other critical pathways requiring further laboratory studies are those associated with beta-adrenergic, cytoskeletal, endocrine, and metabolic diseases. The extracellular matrix (ECM) in GBM is a unique environment that plays an essential role in creating the invasion phenotype and GBM recurrence ${ }^{[40]}$. Some lipid metabolism is related to some properties of GBM, including those involved in the maintenance of cancer stem cells (CSCs) ${ }^{[41]}$. The pathways identified in our analysis may be related to both angiogenesis and the recurrence of GBM.

In GBM, 51 genes are altered in the VEGF signaling pathway (Table 1). However, according to our in silico results, there are other signaling pathways that can indirectly affect VEGF signaling pathway genes expression levels.

\section{Materials And Methods}

\subsection{Study design}

There were 6 main steps based on differentially expressed miRNAs (DEmiRNAs) to find classes of pathways related to the VEGF signaling pathway genes as outlined in the pipeline: data collection including identification of DEmiRNAs based on an expression dataset, finding VEGF signaling pathway genes, detection of associated genes and pathways to VEGF signaling pathway genes, crosstalk between pathways, detection of final related pathways, and class assessment for pathways.

\subsection{Data collection sources}

Data collection was done in two separate sections: finding DEmiRNAs between primary and recurrent GBM patients, and identifying miRNAs involved significantly in the VEGF signaling pathway.

\subsection{Identification of DEmiRNAs}

The miRNA expression datasets of GBM were searched using the keywords: 'GBM', 'recurrent' 'Homo sapiens' [porgn: txid9606]', and 'Expression profiling by array' against the Gene Expression Omnibus (GEO) database (http://www.ncbi.nlm.nih.gov/geo). After a systematic review, one GSE profile (GSE32466) was selected and analyzed. GSE32466 related to miRNA expression was based on GPL10850 (Agilent-021827 Human miRNA Microarray (V3) (miRBase release 12.0 miRNA ID version)).

To identify DEmiRNAs, the GSE32466 dataset was analyzed by Limma R package in Bioconductor that was utilized to mine statistically significant DEmiRNAs based on the difference in their expression values 
between samples of the GBM and recurrent GBM. Significant differential expression was determined as a log2 fold change $\geq|1|$ and the adjusted $p$-value threshold of $0.05^{[42]}$.

\subsection{Definition of VEGF signaling pathway genes}

In the other section, all genes involved in the VEGF signaling pathway come from The Kyoto Encyclopedia of Genes and Genomes (KEGG) pathway database (https://www.genome.jp/kegg/pathway.html). The KEGG is an information base for the networks of genes and metabolic compounds. The expression levels of these genes in GBM were obtained using the Expression Atlas site (www.ebi.ac.uk/gxa).

\subsection{Pathway detection for DEmiRNAs}

DEmiRNAs are essential regulators in many biological pathways. To show the roles of each DEmiRNAs and learn more about their functions, DIANA-miRPath v3.0 (http://www.microrna.gr/miRPathv3) was used. DIANA-miRPath is an online tool that integrates miRNAs with KEGG pathways to provide a deeper understanding of the process by which biological pathways are regulated ${ }^{[43]}$. The threshold $p$-value of 0.05 was considered significant.

\subsection{Identification of genes and pathways related to VEGF signaling pathway}

The genes which had genetic interaction with the VEGF signaling pathway genes were obtained by NetworkAnalyst (https://www.networkanalyst.ca/) based on STRING interactome. They showed medium (400) to high (1000) confidence score. The confidence score cutoff was set at 900 and required experimental evidence. Then, pathways detection was performed for interactional genes using the ToppGene tool (https://toppgene.cchmc.org/), and the adjusted p-value cutoff of 0.05 was considered significant. Moreover, by NetworkAnalyst, all pathways that shared part of the VEGF signaling pathway genes were collected with an adjusted $p$-value $\leq 0.05$. The obtained pathways in the above steps were based on the KEGG.

Furthermore, the Expression Atlas database was used (https://www.ebi.ac.uk/gxa/home) to find dysregulated gene expression of the VEGF signaling pathway genes in GBM.

\subsection{Crosstalk between Pathways}

XTalkDB (http://www.xtalkdb.org) was used for detection of crosstalk between specific pairs of signaling pathways; crosstalk information has been illustrated based on scientific literature [44]. "VEGF signaling pathway" name was inserted into the XTalkDB to find crosstalking pathway pairs. Finally, we got a list of pathways that have inhibitory or activating effects (or both) on the VEGF signaling pathway. On the other hand, the inhibitory or activating effects of the VEGF signaling pathway on other pathways were also obtained.

\subsection{Determination of common miRNAs between VEGF pathway and DE-miRNAs}


MiRNAs related to the VEGF signaling pathway were extracted from the miRWalk3.0 database (http://mirwalk.umm.uni-heidelberg.de/). These miRNAs were compared by the Venny 2.1 free online tool (https://bioinfogp.cnb.csic.es/tools/venny/). This tool was used to find the same miRNAs between DEmiRNAs obtained from the Limma R package and those which involved in the VEGF signaling pathway.

\subsection{Detection of final pathways and class assessment}

To deduce commonalities across the collected pathways, the Venny 2.1 free online tool (https://bioinfogp.cnb.csic.es/tools/venny/) was used. At last, classes of final pathways were determined by the KEGG database.

\section{Conclusion}

Due to the complexity of the biological processes involved in cancers, multiple targeting is very effective in preventing and controlling cancer. Although the VEGF pathway is the most important pathway in angiogenesis process, there are complicated processes in GBM which prevent effective GBM therapy using only VEGF targeting. Our study showed that several pathways or classes might significantly affect the VEGF pathway. Hence, It seems necessary to achieve new targets for combination therapies as well as personal therapies for GBM.

\section{Abbreviations}

VEGF, vascular endothelial growth factor; MAPK, mitogen-activated protein kinase; CNS, central nervous system; IL, Interleukin; TGF-beta, Transforming growth factor beta; TNF, tumor necrosis factor; mTOR, mechanistic target of rapamycin; PI3K, Phosphoinositide 3-kinases; FOXO, forkhead box O; HIF, Hypoxiainducible factors; AMPK, AMP-activated protein kinase; NF-KB, Nuclear factor kappa B; HTLV-1, Human T lymphotropic virus type 1; TCF3, Transcription Factor 3; LEF-1, Lymphoid Enhancer Binding Factor 1; HGF, Hepatocyte Growth Factor; c-MET, mesenchymal-epithelial transition factor; JAK2, Janus kinase 2; STAT3, Signal transducer and activator of transcription 3; UBE3C, Ubiquitin-protein ligase E3C

\section{Declarations}

\section{Acknowledgments}

The authors thank the staff of Cellular and Molecular Research Center and research deputy of Shahrekord University of Medical Scienses and Kerman University of Medical Sciences. We also thank Mr. Roohallah Mahdi for his help on this study. Figure 1 was provided by the pheatmap package v1.0.12 https://cran.r-project.org/web/packages/pheatmap/index.html. Figure 2 was provided by the Cytoscape v3.8.2https://cytoscape.org/. Figure 3 was provided by the Microsoft office v2016https://www.office.com/. 


\section{Authors contributions}

S.G., designed the concept. M.H., analyzed the data. M.H. S.G., and R.S. wrote the main manuscript text. T.L., and M.R.M., have guided and edited the manuscript. M.S., prepared supplementary files. All authors reviewed the manuscript.

\section{Conflict of interest}

The authors declare no potential conflicts of interest to disclose.

\section{Funding source:}

This project was supported by a research grant (Grant No.5750) from Shahrekord University of Medical Sciences IR.SKUMS.REC.1399.282.

\section{References}

1. Alavian, F. \& Ghasemi, S. The Effectiveness of Nanoparticles on Gene Therapy for Glioblastoma Cells Apoptosis: A Systematic Review. Current gene therapy, https://doi.org/10.2174/1566523221666210224110454 (2021).

2. Qian, Z. et al. Differentiation of glioblastoma from solitary brain metastases using radiomic machine-learning classifiers. Cancer Lett, 451, 128-135 (2019).

3. Hundsberger, T., Reardon, D. A. \& Wen, P. Y. Angiogenesis inhibitors in tackling recurrent glioblastoma. Expert Review of Anticancer Therapy, 17, 507-515 (2017).

4. Ahir, B. K., Engelhard, H. H. \& Lakka, S. S. Tumor development and angiogenesis in adult brain tumor: Glioblastoma.Molecular neurobiology,1-18(2020).

5. Testa, U., Pelosi, E. \& Castelli, G. Endothelial progenitors in the tumor microenvironment. Tumor Microenvironment,85-115(2020).

6. Guarnaccia, L. et al. Angiogenesis in human brain tumors: screening of drug response through a patient-specific cell platform for personalized therapy. Scientific reports, 8, 1-13 (2018).

7. Wang, S. et al. FOXF1 promotes angiogenesis and accelerates bevacizumab resistance in colorectal cancer by transcriptionally activating VEGFA. Cancer letters, 439, 78-90 (2018).

8. Ferreira, W. A. S. et al. An update on the epigenetics of glioblastomas. Epigenomics, 8, 1289-1305 (2016).

9. Banelli, B. et al. MicroRNA in glioblastoma: An overview. International journal of genomics 2017 (2017).

10. Shea, A. et al. MicroRNAs in glioblastoma multiforme pathogenesis and therapeutics. Cancer medicine, 5, 1917-1946 (2016).

11. Ghasemi, S. Cancer's epigenetic drugs: where are they in the cancer medicines? The pharmacogenomics journal, 20, 367-379 (2020). 
12. Ghafouri-Fard, S. et al. Regulatory role of microRNAs on PTEN signaling. Biomed. Pharmacother, 133, 110986 (2021).

13. Bo, L. et al. Bioinformatics analysis of miRNA expression profile between primary and recurrent glioblastoma. Eur Rev Med Pharmacol Sci, 19, 3579-3586 (2015).

14. Stackhouse, C. T., Gillespie, G. Y. \& Willey, C. D. Exploring the Roles of IncRNAs in GBM Pathophysiology and Their Therapeutic Potential., 9, 2369 (2020).

15. Angara, K., Borin, T. F. \& Arbab, A. S. Vascular mimicry: a novel neovascularization mechanism driving anti-angiogenic therapy (AAT) resistance in glioblastoma. Translational oncology, 10, 650660 (2017).

16. Burghardt, I. et al. Endoglin and TGF- $\beta$ signaling in glioblastoma.Cell and Tissue Research,112(2021).

17. Sui, H. et al. Tanshinone IIA inhibits $\beta$-catenin/VEGF-mediated angiogenesis by targeting TGF- $\beta 1$ in normoxic and HIF-1a in hypoxic microenvironments in human colorectal cancer. Cancer letters, 403, 86-97 (2017).

18. Gerritsen, M. E., Tomlinson, J. E., Zlot, C., Ziman, M. \& Hwang, S. Using gene expression profiling to identify the molecular basis of the synergistic actions of hepatocyte growth factor and vascular endothelial growth factor in human endothelial cells. British journal of pharmacology, 140, 595-610 (2003).

19. You, W. K. \& McDonald, D. M. The hepatocyte growth factor/c-Met signaling pathway as a therapeutic target to inhibit angiogenesis. BMB reports, 41, 833 (2008).

20. Becker, A. P., Sells, B. E., Haque, S. J. \& Chakravarti, A. Tumor Heterogeneity in Glioblastomas: From Light Microscopy to Molecular Pathology. Cancers, 13, 761 (2021).

21. Qazi, M. et al. Intratumoral heterogeneity: pathways to treatment resistance and relapse in human glioblastoma. Annals of Oncology, 28, 1448-1456 (2017).

22. Zhang, Z. H. et al. Convallatoxin promotes apoptosis and inhibits proliferation and angiogenesis through crosstalk between JAK2/STAT3 (T705) and mTOR/STAT3 (S727) signaling pathways in colorectal cancer., 68, 153172 (2020).

23. Zhang, B., Wang, D., Ji, T. F., Shi, L. \& Yu, J. L. Overexpression of IncRNA ANRIL up-regulates VEGF expression and promotes angiogenesis of diabetes mellitus combined with cerebral infarction by activating NF-KB signaling pathway in a rat model. Oncotarget, 8, 17347 (2017).

24. Soares, R. et al. Evidence for the notch signaling pathway on the role of estrogen in angiogenesis. Molecular endocrinology, 18, 2333-2343 (2004).

25. Nabors, L. B. et al. Tumor necrosis factor a induces angiogenic factor up-regulation in malignant glioma cells: a role for RNA stabilization and HuR. Cancer Res, 63, 4181-4187 (2003).

26. Tomiyama, A. \& Ichimura, K. in Seminars in cancer biology.118-129(Elsevier).

27. Mao, H., LeBrun, D. G., Yang, J., Zhu, V. F. \& Li, M. Deregulated signaling pathways in glioblastoma multiforme: molecular mechanisms and therapeutic targets. Cancer investigation, 30, 48-56 (2012). 
28. Zhang, Y. W., Su, Y., Volpert, O. V. \& Woude, G. F. V. Hepatocyte growth factor/scatter factor mediates angiogenesis through positive VEGF and negative thrombospondin 1 regulation. Proceedings of the National Academy of Sciences 100, 12718-12723(2003).

29. Gong, Y. et al. Insulin-mediated signaling promotes proliferation and survival of glioblastoma through Akt activation. Neuro-oncology, 18, 48-57 (2015).

30. Costa, L. E. et al. Evidence of aquaporin 4 regulation by thyroid hormone during mouse brain development and in cultured human glioblastoma multiforme cells. Frontiers in neuroscience, 13, 317 (2019).

31. Asad, A. S. et al. Prolactin and its receptor as therapeutic targets in glioblastoma multiforme. Scientific reports, 9, 1-16 (2019).

32. Kabat, G. C., Etgen, A. M. \& Rohan, T. E. Do steroid hormones play a role in the etiology of glioma? Cancer Epidemiology and Prevention Biomarkers, 19, 2421-2427 (2010).

33. Panza, S. et al. Leptin and Notch Signaling Cooperate in Sustaining Glioblastoma Multiforme Progression. Biomolecules, 10, 886 (2020).

34. McFaline-Figueroa, J. R. \& Wen, P. Y. The viral connection to glioblastoma. Current infectious disease reports, 19, 5 (2017).

35. Chepied, A. et al. Involvement of the gap junction protein, connexin43, in the formation and function of invadopodia in the human U251 glioblastoma cell line., 9, 117 (2020).

36. Corsaro, A. et al. Cellular prion protein controls stem cell-like properties of human glioblastoma tumor-initiating cells. Oncotarget, 7, 38638 (2016).

37. Stockmann, C., Schadendorf, D., Klose, R. \& Helfrich, I. The impact of the immune system on tumor: angiogenesis and vascular remodeling. Frontiers in oncology, 4, 69 (2014).

38. Brown, N. F., Carter, T. J., Ottaviani, D. \& Mulholland, P. Harnessing the immune system in glioblastoma. British journal of cancer, 119, 1171-1181 (2018).

39. Pan, S. J. et al. Ubiquitin-protein ligase E3C promotes glioma progression by mediating the ubiquitination and degrading of Annexin A7. Scientific reports, 5, 1-11 (2015).

40. Hatoum, A., Mohammed, R. \& Zakieh, O. The unique invasiveness of glioblastoma and possible drug targets on extracellular matrix. Cancer management and research, 11, 1843 (2019).

41. Sun, P. et al. Lipid metabolism enzyme ACSVL3 supports glioblastoma stem cell maintenance and tumorigenicity. BMC cancer, 14, 1-11 (2014).

42. Ritchie, M. E. et al. limma powers differential expression analyses for RNA-sequencing and microarray studies. Nucleic acids research, 43, e47-e47 (2015).

43. Hamzeiy, H. et al. Visualization and Analysis of MicroRNAs within KEGG Pathways using VANESA. Journal of integrative bioinformatics14 (2017).

44. Sam, S. A., Teel, J., Tegge, A. N., Bharadwaj, A. \& Murali, T. XTalkDB: a database of signaling pathway crosstalk. Nucleic acids research, 45, D432-D439 (2017). 
45. Patel-Hett, S. \& D'Amore, P. A. Signal transduction in vasculogenesis and developmental angiogenesis. The International journal of developmental biology, 55, 353 (2011).

46. Kim, J. Y. et al. Second primary cancers in long-term survivors of glioblastoma. Neuro-oncology practice, 6, 386-391 (2019).

47. GUPTA, M. \& BARNETT, G. H. Propylthiouracil-induced Chemical Hypothyroidism with High-dose Tamoxifen Prolongs Survival in Recurrent High Grade Glioma: A. Anticancer Res, 23, 617-626 (2003).

48. Zatkova, M. et al. Projection length stimulated by oxytocin is modulated by the inhibition of calcium signaling in U-87MG cells. Journal of Neural Transmission, 125, 1847-1856 (2018).

49. Takahashi, K. et al. Sialidase NEU3 defines invasive potential of human glioblastoma cells by regulating calpain-mediated proteolysis of focal adhesion proteins. Biochimica et Biophysica Acta (BBA)-General Subjects, 1861, 2778-2788 (2017).

50. Chen, Z., Morales, J. E., Guerrero, P. A., Sun, H. \& McCarty, J. H. PTPN12/PTP-PEST regulates phosphorylation-dependent ubiquitination and stability of focal adhesion substrates in invasive glioblastoma cells. Cancer research, 78, 3809-3822 (2018).

51. Zeng, L. et al. The adherens junction-associated protein 1 is a negative transcriptional regulator of MAGEA2, which potentiates temozolomide-induced apoptosis in GBM. International journal of oncology, 44, 1243-1251 (2014).

52. Valdés-Rives, S. A., Casique-Aguirre, D., Germán-Castelán, L. \& Velasco-Velázquez, M. A. GonzálezArenas, A. Apoptotic signaling pathways in glioblastoma and therapeutic implications. BioMed research international 2017 (2017).

53. Tachon, G. et al. Cell cycle changes after glioblastoma stem cell irradiation: The major role of RAD51. International journal of molecular sciences, 19, 3018 (2018).

54. Zhang, Y. et al. The p53 pathway in glioblastoma. Cancers, 10, 297 (2018).

55. Linher-Melville, K. \& Li, J. The roles of glial cell line-derived neurotrophic factor, brain-derived neurotrophic factor and nerve growth factor during the final stage of folliculogenesis: a focus on oocyte maturation., 145, R43-R54 (2013).

56. Xiong, J. et al. Mature brain-derived neurotrophic factor and its receptor TrkB are upregulated in human glioma tissues. Oncology letters, 10, 223-227 (2015).

57. Tong, B., Pantazopoulou, V., Johansson, E. \& Pietras, A. The p75 neurotrophin receptor enhances HIFdependent signaling in glioma. Experimental cell research, 371, 122-129 (2018).

58. Mainio, A. et al. Decreased quality of life and depression as predictors for shorter survival among patients with low-grade gliomas: a follow-up from 1990 to 2003. European archives of psychiatry and clinical neuroscience, 256, 516-521 (2006).

59. Iwata, N., Ochiai, K., Hayashi, K., Ohashi, K. \& Umemura, T. Nonsuppurative myocarditis associated with so-called fowl glioma. Journal of veterinary medical science, 64, 395-399 (2002). 
60. Kunapuli, P., Lo, K., Hawthorn, L. \& Cowell, J. K. Reexpression of LGI1 in glioma cells results in dysregulation of genes implicated in the canonical axon guidance pathway., 95, 93-100 (2010).

61. Lin, H. Y. et al. CYP17A1 maintains the survival of glioblastomas by regulating SAR1-mediated endoplasmic reticulum health and redox homeostasis. Cancers, 11, 1378 (2019).

62. Pucer, A. et al. Differential role of cathepsins $B$ and $L$ in autophagy-associated cell death induced by arsenic trioxide in U87 human glioblastoma cells. Biological chemistry, 391, 519-531 (2010).

63. Blandin, A. F. et al. Gefitinib induces EGFR and a5 $\beta 1$ integrin co-endocytosis in glioblastoma cells. Cellular and Molecular Life Sciences,1-14(2020).

64. Avci, N. G. et al. NF-kB inhibitor with Temozolomide results in significant apoptosis in glioblastoma via the NF-kB (p65) and actin cytoskeleton regulatory pathways. Sci. Rep, 10, 1-14 (2020).

65. Kilarski, W. W., Jura, N. \& Gerwins, P. Inactivation of Src family kinases inhibits angiogenesis in vivo: implications for a mechanism involving organization of the actin cytoskeleton. Experimental cell research, 291, 70-82 (2003).

66. He, J. J. et al. Activation of $\beta$-adrenergic receptor promotes cellular proliferation in human glioblastoma. Oncology letters, 14, 3846-3852 (2017).

\section{Figures}




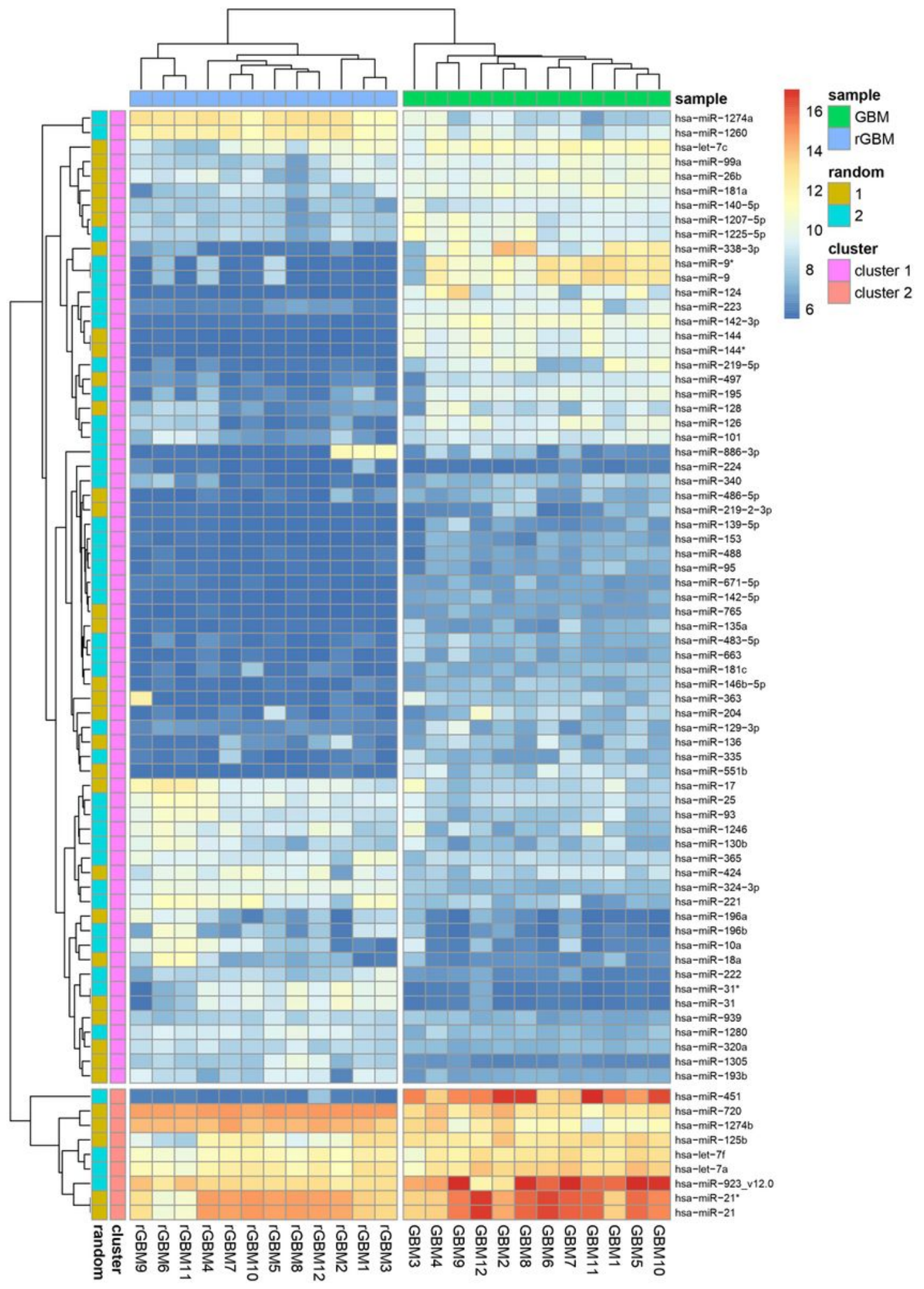

Figure 1

The heatmap corresponding to the hierarchical clustering analysis was performed using the pheatmap function. The columns are samples and the rows are DEmRNAs. The green represents GBM, while blue represents rGBM that are presented above the horizontal axis. 


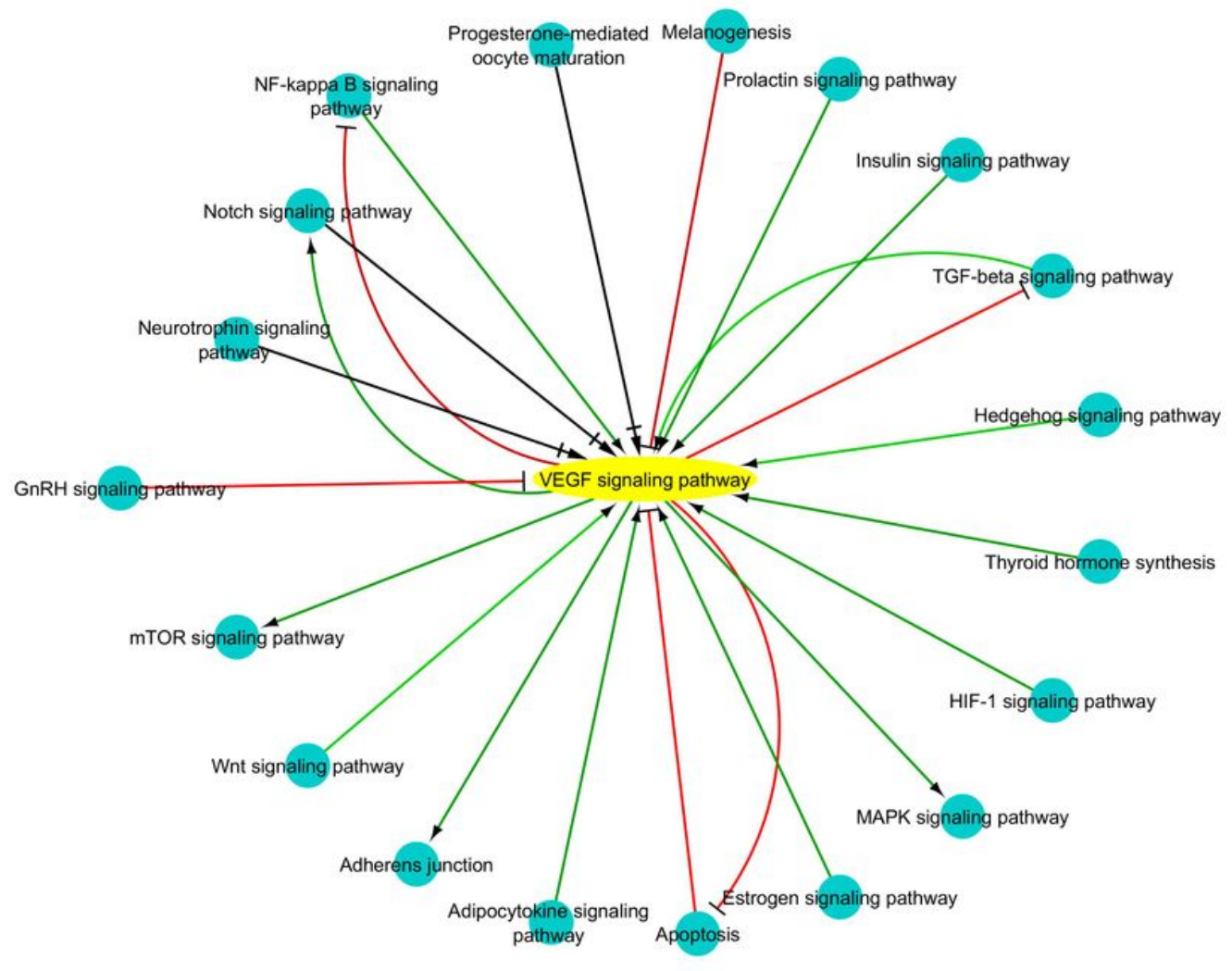

Figure 2

Crosstalk between VEGF signaling pathways and other pathways: Inhibition, activation and dual effects have been represented by red, green and black edges respectively. 
a Signal transduction

= Cancer: specific types

Endocrine system

a Cancer: overview

- Infectious disease: bacterial

- Infectious disease: viral

- Cellular community - eukaryotes

a Cell growth and death

- Neurodegenerative disease

ammune system

E Nervous system

- Cardiovascular disease

= Development and regeneration

= Folding, sorting and degradation

= Transport and catabolism

= Infectious disease: parasitic

a Circulatory system

Endocrine and metabolic disease

Excretory system

= Signaling molecules and interaction

n Lipid metabolism

= Translation

- Environmental adaptation

Digestive system

w Cell motility

E Carbohydrate metabolism

= Substance dependence

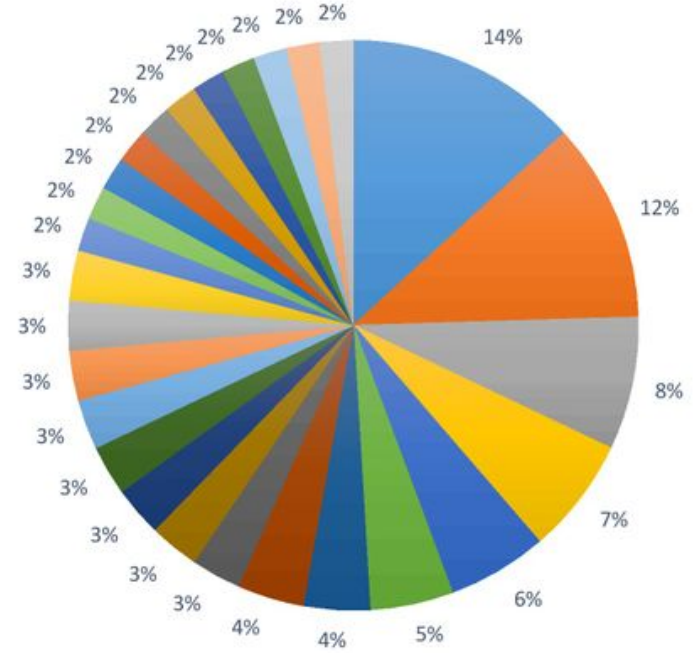

\section{Figure 3}

Classification of all finalized pathways: This percentage is sorted by the number of pathways associated with each class. The percentages here are related to the variety of pathways in each class.

\section{Supplementary Files}


This is a list of supplementary files associated with this preprint. Click to download.

- SupplementaryFile1.xlsx

- Graphicalabstract.pdf16001.tiff

- SupplementaryFile3.xIsx

- SupplementaryFile4.xlsx

- supplementaryfile6.docx

- supplementaryfile7.xlsx 